\title{
Exploiting machine learning for predicting skeletal-related events in cancer patients with bone metastases
}

\author{
Zhiyu Wang ${ }^{1, *}$, Xiaoting Wen ${ }^{1,}{ }^{*}$, Yaohong Lu ${ }^{1}$, Yang Yao ${ }^{1}$, Hui Zhao ${ }^{1}$ \\ ${ }^{1}$ Department of Internal Oncology, Shanghai Jiao Tong University Affiliated Sixth People's Hospital, Shanghai, China \\ *These authors contributed equally to this work
}

Correspondence to: Hui Zhao, e-mail: zhao-hui@sjtu.edu.cn

Keywords: machine learning, skeletal-related events, bone metastases, decision tree, support vector machine

Received: October 11, 2015

Accepted: January 24, 2016

Published: February 09, 2016

\section{ABSTRACT}

The aim of the bone metastases (BM) treatment is to prevent the occurrence of skeletal-related events (SREs). In clinical, physicians could only predict the occurrence of SREs by subjective experience. Machine learning (ML) could be used as predictive models in the medical field. But there is no published research using ML to predict SREs in cancer patients with BM. The purpose of this study was to assess the associations of clinical variables with the occurrence of SREs and to subsequently develop prediction models to help identify SREs risk groups.

We analyzed 1143 cancer patients with BM. We used the statistical package of SPSS and SPSS Modeler for data analysis and the development of the prediction model. We compared the performance of logistic regression (LR), decision tree (DT) and support vector machine(SVM). The results suggested that Visual Analog Scale (VAS) scale was a key factor to SREs in LR, DT and SVM model. Modifiable factors such as Frankel classification, Mirels score, $\mathrm{Ca}$, aminoterminal propeptide of type I collagen (PINP) and bone-specific alkaline phosphatase (BALP) were identified. We found that the result of applying LR, DT and SVM classification accuracy was $79.2 \%$, $85.8 \%$ and $88.2 \%$, with 9,4 and 8 variables, respectively.

In conclusion, DT and SVM achieved higher accuracies with smaller number of variables than the number of variables used in LR. ML techniques can be used to build model to predict SREs in cancer patients with BM.

\section{INTRODUCTION}

Bone is the most common site of metastasis in cancer. Cancer metastases to the bone are most prevalent among patients with advanced cancer of the breast (73\%), prostate (68\%), or lung (36\%) [1]. Bone metastases (BM) can lead to skeletal-related events (SREs), defined as pathologic fracture, spinal cord compression, requirement for radiation, surgery to bone, and hypercalcemia [2-7]. Data from the untreated arms of clinical trials indicates that SREs are most common in patients with BM secondary to breast cancer (2-year cumulative incidence of $68 \%$ ), followed by prostate cancer (2-year cumulative incidence of $49 \%$ ), and non-small cell lung cancer (NSCLC) and other solid tumors (OST) (21 month cumulative incidence of 48\%) [8-10]. Observational studies yielded similar patterns, with a 1-year cumulative incidence of SREs after
BM diagnosis of $46 \%$ in prostate cancer patients and $38 \%$ in female breast cancer patients $[11,12]$.

BM and subsequent SREs can be an important burden on a cancer patients' quality of life (QOL) and overall health status [13, 14]. SREs will dramatically reduce patients' QOL and even shorten survival [15]. The aim of the BM treatment is to prevent the occurrence of SREs. Many risk factors play important roles in the incidence of SREs. But in clinical, physicians could only predict the occurrence of SREs by subjective experience. Multidimensional analysis of SREs requires considerable effort and expertise, demanding the development of more sophisticated ways to facilitate such complex, preferably automatic analysis [16].

Machine learning (ML) utilizes a variety of artificial intelligence and statistical models to learn from the observed data in order to create reasonable generalizations, discover patterns, classify previously unseen data or 
predict new directions [17]. The medical field is quickly embracing ML methodologies, such as decision tree (DT), support vector machine (SVM), as these approaches have shown progress in their usefulness in prediction and classification. Predictive models are used in a variety of medical domains for diagnostic and prognostic tasks. An increasingly large number of medical data are collected routinely, and often automatically, in many areas of medicine [18]. This implementation could prove useful in discovering ways to lower the cost of medication, improve clinical studies and help facilitate better assessments by physicians [19]. It is a opportunity for the field of ML and statistics to extract useful information and knowledge from this wealth of data [18].

ML has been used in the medical field to diagnose lung cancer, breast cancer, asthma, heart disease, dementia and other diseases and conditions. But there is no published research using supervised ML to predict SREs in cancer patients with BM. The purposes of this study were to identify the factors influencing SREs, to compare the accuracy of logistic regression(LR)-, DT- and SVM-based models in predicting SREs and to develop an effective and efficient model to predict SREs in cancer patients with $\mathrm{BM}$ that require intervention, based on laboratory tests commonly performed in clinical practice.

\section{RESULTS}

\section{General characteristics of patients}

Our study included 1143 patients with BM, median followup time was approximately 7 months. Table 2 shows the socio-demographic and clinical characteristics of the patients.

622 patients $(54.4 \%)$ had SREs, 284 patients (24.8\%) had developed multiple SREs, 263 patients $(23.0 \%)$ had prior SREs. The rank one SREs were radiation to bone, following by pathological fracture and surgery to bone (Table 3 ). Most of patients suffered from lung cancer, following by breast cancer, cancer of unknown primary and prostate cancer (Table 4).

\section{Development of the LR model}

Taking into account the 9 variables, the results of applying LR accuracy was 79.2\%. VAS scale, Frankel classification, Mirels score, Gender, Cancer type, Ca, PINP, $\beta$-CTX and BALP are selected as significant variables in the LR model. A complete list of study variables in each variable set along with $p$-values are listed in Table 5.

\section{Development of the DT model}

Figure 1 shows DT classification of SREs in patients with BM. The DT classification of SREs consisted of
4 variables which in order of importance were the following: VAS scale, PINP, CA 153 and BALP. In Figure 1 , each node shows the probability of SREs for patients with BM whom are satisfied in mentioned conditions in corresponding branches.

We used CART analysis to explore high-order interactions between variables. For example, CART analysis exhibited VAS scale is the most important factor affecting SREs in patients with BM. In individuals with VAS scale Grade 3, there is an interaction with CA153, while with VAS scale Grade 1 or 2, there is an interaction with PINP, and in patients with PINP $\geq 101.8 \mathrm{ng} / \mathrm{ml}$, there is an interaction with BALP. Figure 1 shows interactions between variables clearly.

\section{Development of the SVM model}

To identify the variables that had the highest classification accuracy in prediction of SREs, we used SVM with radial basis function (parameter $\mathrm{C}=1$, $\gamma=1 /$ number of features) that systematically searched through the space of subsets of variables, and evaluated the goodness of each variable subset according to the prediction accuracy. The variable subset showing the highest accuracy was identified as the predictor set. Parameter $\mathrm{C}$ is the weights between empirical error and generalization error. Parameter $\gamma$ controls the shape of the separating hyperplane. It was similar to previous study on predictors of medication adherence in elderly patients with chronic disease [24].

We listed the top 8 ranked variables selected by SVM and their prediction accuracies using a combination of the top ranked variables together in Table 6 to examine the above results in detail. The accuracy using a single variable selected as VAS scale was $55.1 \%$. The present accuracy of the SVM reached $67.4 \%$ with two variables, VAS scale and Frankel classification. The highest accuracy, 97.1\%, was achieved with eight predictors: VAS scale, Frankel classification, Ca, Cancer type, Gender, Mirels score, PINP and Character of BM. The performance was very markedly decreased when more than 8 features were selected. Unlike the intuition that having more variables should achieve higher predictive performance, we found that using a small number of variables can achieve higher prediction accuracy.

\section{Comparison among prediction models}

Table 7 compares the experimental results of LR, DT and SVM using 5 evaluation measures. SVM and DT showed better performance than LR in overall scoring categories, allowing identification of predictor candidates to determine the most probable SREs of a patient.

LR showed 79.2\% accuracy when all 9 variables were used for the prediction of SREs (Tables 5 and 7). Compared to the result of LR, the result of DT showed 
Table 1: Summary of attributes included in the study

\begin{tabular}{|c|c|}
\hline Attribute & Represented as \{permissible value\} \\
\hline Gender & Categorical $\{$ Male (1) / Female (2) \} \\
\hline Age & Numeric \\
\hline KPS Scale & Categorical $\{\geq 70(1) /<60(2)\}$ \\
\hline VAS scale & Categorical $\{\text { Grade } 1(1) / 2(2) / 3(3)\}^{*}$ \\
\hline Character of BM & Categorical \{ Lytic (1) / Blastic (2) / Mixed (3) \} \\
\hline The extent of BM & Categorical $\{\text { Soloway } 1(1) / 2(2) / 3-4(3)\}^{* *}$ \\
\hline Visceral metastases & Categorical $\{\text { Without }(1) / \text { With }(2)\}^{* * *}$ \\
\hline Frankel classification & Categorical $\{\text { A (1) / B (2) / C (3) / D (4) / E (5) / Without spine metastasis (6) }\}^{* * * *}$ \\
\hline Mirels scale & Categorical $\{$ Without extremity metastasis $(0) / 4-6(1) / 7-9(2) / 10-12(3)\} * * * * *$ \\
\hline$\beta-\mathrm{CTX}$ & Numeric \\
\hline N-MID & Numeric \\
\hline PINP & Numeric \\
\hline BALP & Numeric \\
\hline CEA & Numeric \\
\hline CA125 & Numeric \\
\hline CA153 & Numeric \\
\hline CA199 & Numeric \\
\hline $\mathrm{AKP}$ & Numeric \\
\hline $\mathrm{Ca}$ & Categorical $\{\geq 2.6 \mathrm{mmol} / 1(1) /<2.6 \mathrm{mmol} / 1$ (2) $\}$ \\
\hline
\end{tabular}

*Pain level on a 10-point scale, with 0 representing no pain and 10 representing the maximum pain intensity imaginable. Grade 1: 0-3, Grade 2: 4-6, Grade 3: 7-10.

**Soloway 0 refers to patients without BM; Soloway 1 refers to patients with $<6 \mathrm{BM}$; Soloway 2 refers to patients with $<20$ BM; Soloway 3 refers to patients with $>20$ but less than a "super scan"; Soloway 4 refers to patients with "super scan" that is defined by a $>75 \%$ involvement of the ribs, vertebrae, and pelvic bones [19].

***Visceral metastases defined as distant metastases, except for BM, including brain metastases.

****Frankel classification of spinal cord injury [20]: Class A representing complete paralysis, Class B representing sensory function only below the injury level, Class $\mathrm{C}$ representing incomplete motor function below injury level, Class D representing fair to good motor function below injury level, and Class E representing normal function.

*****Mirels scoring system [21] based on pain intensity, site, type (lytic, mixed or blastic) and amount of bony involvement.

significantly higher accuracy, $85.8 \%$, with only 4 variables on the same patient samples (Table 7). Compared to the result of LR, the result of SVM showed significantly higher accuracy, $88.2 \%$, with 8 variables on the same patient samples (Table 7). This result indicates that ML techniques (DT and SVM) can achieve greater accuracy with a smaller number of variables than the number of variables used in LR. It is interesting to note that the most significant variable (VAS scale) selected by the DT and SVM agrees with that selected by LR.

The results of the comparison of the discriminatory power of LR, DT and SVM models are summarized in Tables 7 and 8 . The AUC indicates how well a prediction model discriminates between healthy patients and patients with disease. The following guidelines have been proposed for interpretation of this area: $0.5-0.7$, rather low accuracy; 0.7-0.9, moderate accuracies useful for some purposes; and $>0.9$, rather high accuracy [25]. Therefore, the classification accuracies of these models were moderate.

Our results indicated that the DT and SVM model had better diagnostic capability than LR model. The AUC had achieved a moderate diagnostic power.

\section{DISCUSSION}

Health and medical data are exponentially increasing, necessitating various means to take advantage of huge amounts of data. Big data technologies enable the fast processing of massive amounts of data [26]. 
Table 2: Baseline characteristics of patients

\begin{tabular}{|c|c|c|c|}
\hline Variables & All patients & With SREs & Without SREs \\
\hline Gender $(1 / 2)$ & $614 / 529$ & $382 / 240$ & $232 / 289$ \\
\hline Age $($ mean $\pm \mathrm{SD})$ & $59.9 \pm 23.7$ & $58.8 \pm 11.6$ & $61.3 \pm 32.8$ \\
\hline KPS Scale $(1 / 2)$ & $969 / 174$ & $515 / 107$ & $454 / 67$ \\
\hline VAS scale( $(1 / 2 / 3)$ & $270 / 444 / 429$ & $69 / 191 / 362$ & $201 / 253 / 67$ \\
\hline Character of BM $(1 / 2 / 3)$ & $917 / 83 / 143$ & $517 / 34 / 71$ & $400 / 49 / 72$ \\
\hline The extent of BM $(1 / 2 / 3)$ & $178 / 610 / 355$ & 108/316/198 & $70 / 294 / 157$ \\
\hline Visceral metastases $(1 / 2)$ & $623 / 520$ & $347 / 275$ & $276 / 245$ \\
\hline Frankel classification $(1 / 2 / 3 / 3 / 5)^{*}$ & $20 / 20 / 56 / 58 / 843$ & $20 / 19 / 47 / 47 / 418$ & $0 / 1 / 9 / 11 / 425$ \\
\hline Mirels $(1 / 2 / 3)^{* *}$ & $38 / 228 / 25$ & $13 / 132 / 22$ & $25 / 96 / 3$ \\
\hline$\beta$-CTX $($ mean \pm SD $)$ & $772.9 \pm 488.4$ & $810.5 \pm 548.2$ & $741.6 \pm 430.2$ \\
\hline N-MID $($ mean \pm SD) & $22.4 \pm 40.1$ & $24.4 \pm 45.3$ & $20.8 \pm 35.0$ \\
\hline $\mathrm{PINP}($ mean $\pm \mathrm{SD})$ & $166.3 \pm 156.5$ & $195.8 \pm 193.3$ & $141.7 \pm 111.8$ \\
\hline $\mathrm{BALP}($ mean $\pm \mathrm{SD})$ & $35.7 \pm 25.4$ & $35.8 \pm 26.0$ & $35.5 \pm 24.6$ \\
\hline $\mathrm{CEA}($ mean $\pm \mathrm{SD})$ & $173.4 \pm 569.1$ & $203.8 \pm 499.0$ & $148.0 \pm 620.8$ \\
\hline CA125 $($ mean \pm SD $)$ & $171.8 \pm 473.3$ & $195.6 \pm 592.6$ & $143.3 \pm 265.1$ \\
\hline CA153 $($ mean \pm SD $)$ & $91.1 \pm 213.0$ & $91.9 \pm 205.9$ & $90.4 \pm 218.9$ \\
\hline CA199 $($ mean \pm SD $)$ & $299.5 \pm 1034.9$ & $347.0 \pm 1196.2$ & $259.8 \pm 877.3$ \\
\hline $\mathrm{AKP}($ mean $\pm \mathrm{SD})$ & $184.7 \pm 217.0$ & $194.4 \pm 241.5$ & $176.6 \pm 194.0$ \\
\hline $\mathrm{Ca}(1 / 2)$ & $1091 / 52$ & $570 / 52$ & $521 / 0$ \\
\hline
\end{tabular}

*Among 997 patient with spine metastasis, 551 with SREs, 446 without SREs.

**Among 291 patient with extremity metastasis , 167 with SREs, 124 without SREs.

Among these technologies, artificial intelligence has regained prominence as an important tool to provide intelligent services for big data, and ML techniques have also been used extensively for such purposes [27].

Traditional statistical methods, such as LR, have become increasingly difficult to use for prediction models due to several constraints that dictate the low statistical power with small sample size and complex polynomial interaction terms with curvilinear effects among the relationship of variables.

SVM and LR are similar in that both calculate a set of coefficients for variables based on a transformation of the feature space [28]. The major difference between SVM and LR is that while LR attempts to explicitly model the probability (via the odds) of outcomes, SVM attempts to directly find the best dividing hyperplane (or hyperplanes, in the case of more than two classes) regardless of the actual probability of class membership [27]. There are several advantages of SVM compared to LR. While in LR the data analyst must explicitly choose to increase the dimensionality of the feature space through the addition of interaction or polynomial terms among predictors, such transformations are standard practice in SVM approaches to classification [29]. In addition, SVM deals well with high-dimensional data, and they do not assume a parametric relationship between the model predictors and outcome.

DTs are classification algorithms which specify a "tree" of cut points that minimize some measure of diversity in the final nodes once the tree is complete. The final nodes then represent relatively homogenous individual classes [28]. To the extent that all data points classified at a given end node have a similar probability of class membership (that is, probability of treatment), then the output of DTs can be used to directly construct propensity categories [30]. Many methods for DTs (e.g. ID3, C4.5) do not provide a probability of class membership although some variants, in particular CART do provide such probabilities. However, performance of all DTs is dependent on both their method of construction and the amount of pruning (removal of highly specific nodes) performed. The major advantage of DT analysis over LR analysis is that the results of analysis are easy to understand. The simple allocation of patients into subgroups by following the flowchart form could define the predicted possibility of outcome. 
Table 3: Summary of SREs in the study

\begin{tabular}{|c|c|c|}
\hline SREs & People & Times \\
\hline Total confirmed events & 973 & 1123 \\
\hline Radiation to bone & $347(35.7 \%)$ & $464(41.3 \%)$ \\
\hline Pathological fracture & $248(25.4 \%)$ & $263(23.4 \%)$ \\
\hline Spinal cord compression & $97(10.0 \%)$ & $97(8.6 \%)$ \\
\hline Surgery to bone & $229(23.6 \%)$ & $245(21.8 \%)$ \\
\hline Hypercalcemia & $52(5.3 \%)$ & $54(4.9 \%)$ \\
\hline
\end{tabular}

Table 4: Summary of cancer type and SREs in the study

\begin{tabular}{|l|c|c|c|}
\hline \multicolumn{1}{c|}{ Cancer type } & All (\%) & With SREs & Without SREs \\
\hline Lung cancer & $566(49.5 \%)$ & $256(45.2 \%)$ & $310(54.8 \%)$ \\
\hline Breast cancer & $173(15.1 \%)$ & $115(66.5 \%)$ & $58(33.5 \%)$ \\
\hline Prostate cancer & $71(6.2 \%)$ & $37(52.1 \%)$ & $34(47.9 \%)$ \\
\hline Urinary cancer & $56(4.9 \%)$ & $38(67.9 \%)$ & $18(32.1 \%)$ \\
\hline Colorectal cancer & $54(4.7 \%)$ & $32(59.3 \%)$ & $22(40.7 \%)$ \\
\hline Esophagus and stomach cancer & $40(3.5 \%)$ & $20(50.0 \%)$ & $20(50.0 \%)$ \\
\hline Liver cancer & $35(3.1 \%)$ & $29(82.9 \%)$ & $6(17.1 \%)$ \\
\hline Nasopharyngeal cancer & $20(1.7 \%)$ & $12(60.0 \%)$ & $8(40.0 \%)$ \\
\hline Thyroid cancer & $16(1.4 \%)$ & $11(68.8 \%)$ & $5(31.2 \%)$ \\
\hline Gynecologic cancer & $16(1.4 \%)$ & $12(75.0 \%)$ & $4(25.0 \%)$ \\
\hline Cancer of unknown primary & $78(6.8 \%)$ & $46(59.0 \%)$ & $32(41.05)$ \\
\hline Other cancer & $18(1.6 \%)$ & $14(77.8 \%)$ & $4(22.2 \%)$ \\
\hline
\end{tabular}

In this study, ML models based on routinely available clinical and laboratory parameters were constructed for SREs prediction in cancer patients with BM. As expected, ML techniques (DT and SVM) showed greater accuracy with a smaller number of variables than the number of variables used in LR, because they establish the optimal classifier to maximize the geometric margin between samples and therefore minimize empirical classification errors. In this analysis, VAS scale was revealed as the strongest predictor of SREs. VAS scale, PINP, CA153 and BALP were selected as the predictors of SREs according to the DT model. In SVM, VAS scale, Frankel classification, Ca, Cancer type, Gender, Mirels score, PINP and Character of BM were selected as the predictors.

Most of SREs were radiation to bone, and the aim of radiation was to relieve pain. Bone surgery also had the analgesic effect. That maybe the reason why VAS scale was revealed as the strongest predictor of SREs. These findings suggested that providing appropriate analgesic therapy may reduce the occurrence of SREs. Frankel classification was designed for the assess of spinal cord compression, while Mirels score for limbs pathological fracture. These clinical factors were also revealed as the predictor of SREs. These BTMs and tumor markers were not linear with SREs. In DT, patients with moderately elevated PINP, BALP were with the highest proportion of SREs. SREs are complex phenomenons with many causes and correlates. SREs are not only related with bone formation and bone resorption, but also with the sites of $\mathrm{BM}$, soft tissue mass and many other factors. Serum BTMs could only reflect bone formation and bone resorption, that maybe the reason why serum BTMs were not as well as clinical factors in the prediction of SREs.

We found that the occurrence of SREs in our study was higher than some clinical trials. The reason was because we defined percutaneous osteoplasty (PO) as bone surgery. PO can immediately restore the mechanical properties of the affected skeletal segment, provide the treated bones with increased resistance to compressive stresses, and prevent further risk of fractures, allowing immediate weight-bearing. PO can be uesed not only in vertebral metastases, but also in pelvic, iliac, and femoral metastases. PO would be effective as a combined-modality 
Table 5: LR model of SREs in patients with BM

\begin{tabular}{|c|c|c|c|}
\hline Variable & Coefficient & SE & $p$-value \\
\hline \multicolumn{4}{|l|}{ VAS scale } \\
\hline 1 & -3.16 & 0.24 & $<0.01$ \\
\hline 2 & -2.27 & 0.21 & $<0.01$ \\
\hline 3 & -0.43 & 0.18 & $<0.01$ \\
\hline \multicolumn{4}{|c|}{ Mirels score } \\
\hline 1 & -2.58 & 0.89 & 0.24 \\
\hline 2 & -2.57 & 0.97 & 0.41 \\
\hline 3 & -2.43 & 0.89 & 0.31 \\
\hline \multicolumn{4}{|l|}{ Gender } \\
\hline 1 & 1.65 & 0.21 & 0.22 \\
\hline \multicolumn{4}{|c|}{ Cancer type } \\
\hline 1 & -0.01 & 0.82 & 0.46 \\
\hline 2 & 2.67 & 0.85 & 0.17 \\
\hline 3 & -0.04 & 0.87 & 0.96 \\
\hline 4 & 0.19 & 0.89 & 0.83 \\
\hline 5 & 0.48 & 0.88 & 0.58 \\
\hline 6 & 0.66 & 0.97 & 0.49 \\
\hline 7 & 0.93 & 1.00 & 0.35 \\
\hline 8 & -0.33 & 1.03 & 0.75 \\
\hline 9 & 1.31 & 1.10 & 0.23 \\
\hline 10 & 0.97 & 1.09 & 0.37 \\
\hline 11 & 0.01 & 0.88 & 0.98 \\
\hline \multicolumn{4}{|c|}{ Frankel classification } \\
\hline 1 & -5.86 & 0.40 & $<0.01$ \\
\hline 2 & -5.42 & 0.45 & $<0.01$ \\
\hline 3 & -4.25 & 0.37 & $<0.01$ \\
\hline 4 & -4.65 & 0.38 & $<0.01$ \\
\hline 5 & -3.29 & 0.52 & $<0.01$ \\
\hline PINP & -0.01 & 0.01 & 0.07 \\
\hline BALP & 0.03 & 0.02 & 0.36 \\
\hline$\beta-\mathrm{CTx}$ & -0.01 & 0.01 & 0.54 \\
\hline \multicolumn{4}{|l|}{$\mathrm{Ca}$} \\
\hline 1 & -1.21 & 0.05 & 0.02 \\
\hline Constant & 5.74 & 0.92 & 0.63 \\
\hline
\end{tabular}

SE: standard error, CI: confidence interval. 
therapy for the treatment of BM [31]. We observed that suitable bone surgery, bone radiotherapy would not reduce patient's quality of life. This is just the opposite of what we defined in SREs. If there is a large clinical trial results can support this hypothesis, it will have a great impact on this model.

Our study is, to the best of our knowledge, the first attempt to use ML techniques to identify the influencing factors and to apply prediction models for the SREs of the cancer patients with BM as an alternative and complement to the traditional statistical approaches. We only used SPSS and SPSS Modeler to construct all the DT, SVM and LR model. As we all know, SPSS is widely used in the medical field for its user friendly. It would be easier for other physicians to use the models in SPSS than other software. ML models may open new possibilities to find health-related factors that otherwise would be hidden in traditional analysis methods. We used ML techniques as a supplement to the LR to develop prediction models for SREs risk groups. Our study can be used as data in healthcare for the development of new clinical assessment and interventions for the cancer patients with BM. In other words, it would be possible to develop, specifically for the cancer patients with BM, an SREs measurement tool that helps prioritize intervention for SREs risk groups. Based on the identified influencing factors, this study could also provide guidelines for healthcare staff in caring for the cancer patients with BM and could help fine-tune and improve healthcare intervention in practice.

Identification of the risk factors associated with SREs development in cancer patients with BM is essential for formulating personalized surveillance programs. Treatment of BM aims to prevent the incidence of SREs includes orthopedic management, radiation, surgery, and systemic treatments (eg, bone-targeting agents (BTAs), endocrine therapy and chemotherapy). Our Network

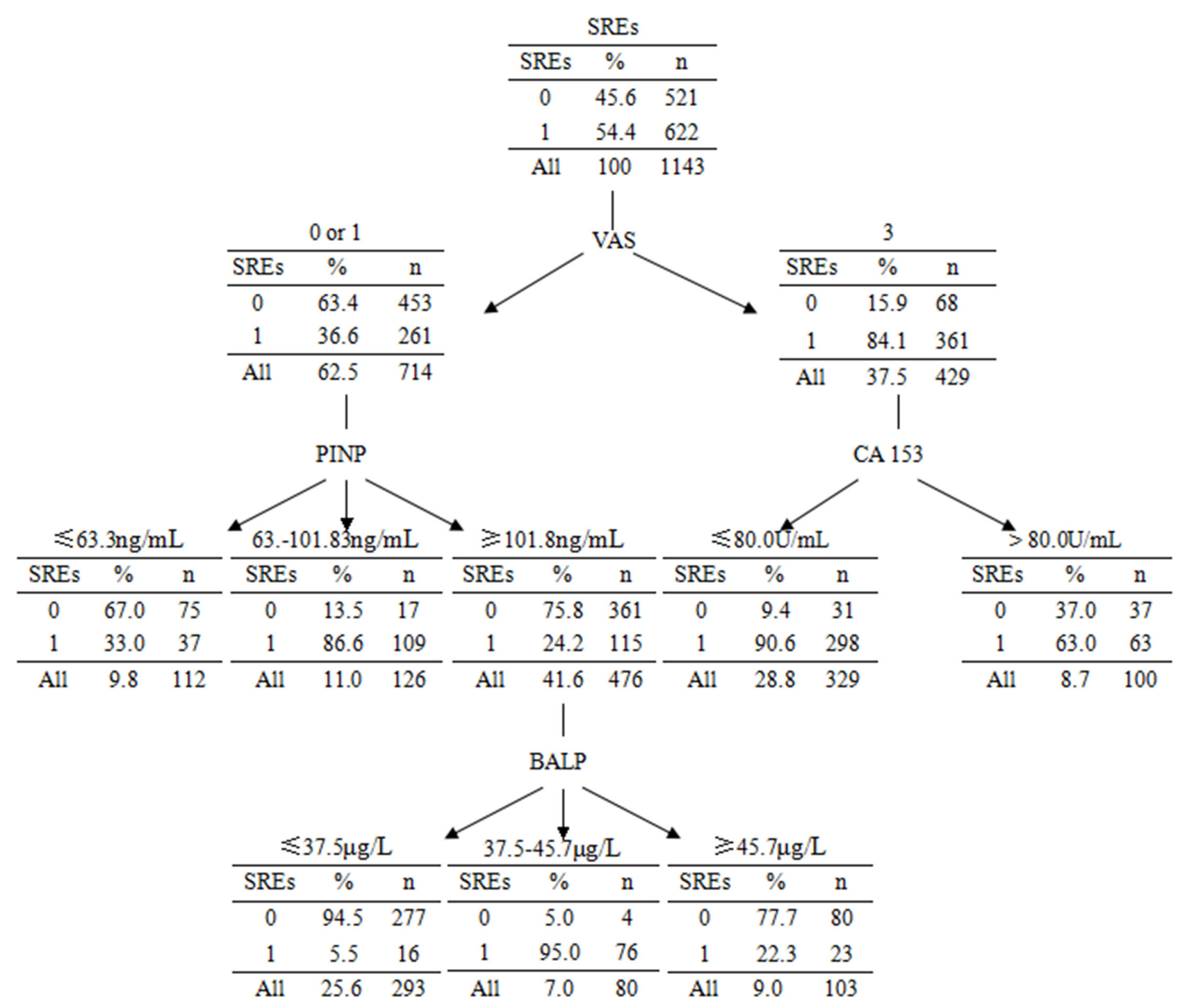

Figure 1: DT model of SREs in patients with BM. 
Table 6: Combination of the top eight variables and classification accuracy in SVM

\begin{tabular}{|c|l|c|}
\hline \multicolumn{1}{|c|}{ No. of variables } & \multicolumn{1}{c|}{ Accuracy (\%) } \\
\hline 1 & VAS scale & 55.1 \\
\hline 2 & VAS scale, Frankel classification & 67.4 \\
\hline 3 & VAS scale, Frankel classification, Ca & 76.6 \\
\hline 4 & VAS scale, Frankel classification, Ca, Cancer type & 84.3 \\
\hline 5 & VAS scale, Frankel classification, Ca, Cancer type, Gender & 91.7 \\
\hline 6 & VAS scale, Frankel classification, Ca, Cancer type, Gender, Mirels score & 94.4 \\
\hline 7 & $\begin{array}{l}\text { VAS scale, Frankel classification, Ca, Cancer type, Gender, Mirels score, } \\
\text { PINP }\end{array}$ & 96.2 \\
\hline 8 & $\begin{array}{l}\text { VAS scale, Frankel classification, Ca, Cancer type, Gender, Mirels score, } \\
\text { PINP, Character of BM }\end{array}$ & 97.1 \\
\hline
\end{tabular}

Table 7: Comparison between LR, DT and SVM

\begin{tabular}{|c|c|c|c|c|c|c|c|}
\hline \multicolumn{1}{c}{ Model } & \multicolumn{2}{c}{$\begin{array}{c}\text { Predicted } \\
\text { positive }\end{array}$} & \multicolumn{2}{c}{$\begin{array}{c}\text { Predicted } \\
\text { negative }\end{array}$} & \multicolumn{2}{c}{$\begin{array}{c}\text { Accuracy } \\
(\%)\end{array}$} & \multicolumn{2}{c}{$\begin{array}{c}\text { Sensitivity } \\
(\%)\end{array}$} & \multicolumn{2}{c}{$\begin{array}{c}\text { Specificity } \\
(\%)\end{array}$} & \multicolumn{2}{c}{ PPV (\%) } & NPV (\%) \\
\hline LR & & & 79.2 & 79.4 & 78.9 & 81.8 & 76.3 \\
\hline Positive & 494 & 128 & & & & & \\
\hline Negative & 110 & 411 & & & & & \\
\hline DT & & & 85.8 & 87.9 & 83.3 & 86.2 & 85.3 \\
\hline Positive & 547 & 75 & & & & & \\
\hline Negative & 87 & 434 & & & & & \\
\hline SVM & & & 88.2 & 88.6 & 87.7 & 89.6 & 86.5 \\
\hline Positive & 551 & 71 & & & & & \\
\hline Negative & 64 & 457 & & & & & \\
\hline
\end{tabular}

PPV: positive predictive value, NPV: negative predictive value.

Table 8: Comparison of AUC between LR, DT and SVM

\begin{tabular}{|c|c|c|c|c|}
\hline \multirow{2}{*}{ Model } & \multirow{2}{*}{ AUC } & \multirow{2}{*}{ SE } & \multicolumn{2}{c}{ 95\% CI for Exp (B) } \\
\cline { 4 - 5 } & & & Lower & Upper \\
\hline LR & 0.792 & 0.012 & 0.767 & 0.815 \\
\hline DT & 0.856 & 0.011 & 0.835 & 0.876 \\
\hline SVM & 0.882 & 0.010 & 0.861 & 0.900 \\
\hline
\end{tabular}

Meta-Analysis showed denosumab, zoledronate and pamidronate were generally effective in preventing SREs in cancer patients with BM and denosumab and zoledronate were also associated with reductions in risk of pathologic fractures and radiation compared to placebo [32]. The research was not finished when these model were found, and the model should included some decision support system. Our models can predict SREs and then direct when and what treatment should be done. With low and medium level, we would give patients BTAs; and for high level, we would give patients orthopedic management, radiation even surgery. After PO, SREs especially pathologic fracture were rarely happened in the treated bone. So PO is highly recommend in the high level patients for its highly effective and safety.

The current study has several limitations, which have to be improved for prospective studies in prediction modeling. 
First, it was limited to examining the impacts of individual variables. We did not examine how each variable affects others; nor did we study the nature of direct or indirect influencing factors. In future studies, we need to study how they affect predictability by identifying the meaning and detailed univariate analysis will be needed.

Second, the classification accuracies of these models were moderate. In our study, the cross validation method used the same data as the test data and the training data. If there are enough samples in the future, we will be able to get more accurate results by ensuring that the test data and the training data are separated in advance.

In this study, we sought to assess the capacity of LR, DT and SVM models to predict SREs, with the goal of developing a more predictive profile for identifying important clinical risk facts that affect SREs recurrence. We found that ML served as an effective alternative to conventional LR in identifying the key variables to show the higher classification accuracy, thereby created valuable diagnostic programs for SREs prediction.

\section{MATERIALS AND METHODS}

\section{Data collection}

This cross sectional retrospective study enrolled 1143 cancer patients with BM of both sexes, recruited from Department of Internal Oncology, Shanghai Sixth People's Hospital in the period between June 2007 and June 2014. This study was approved by the ethics committee of the Sixth People's Hospital, Shanghai Jiao Tong University. The principles of the Declaration of Helsinki were followed. Written consent was obtained. The diagnosis of cancer had been made by using the standard clinical criteria.

\section{Feature selection and reduction}

A subset of 19 features including routine laboratory workup (categorical or numerical) was used for the model building process (Table 1). The dataset was created containing 2 demographic variables (age, gender), 2 general conditional variables $\{$ Karnofsky Performance Scale (KPS) and Visual Analog Scale (VAS) \}, 3 metastases variables (Character of BM, extent of BM and Visceral metastases), 2 injured variables (Frankel classification of spinal cord injury and Mirels scale), 4 bone turnover markers(BTM) \{bone-specific alkaline phosphatase (BALP), N-terminal midfragment of osteocalcin (N-MID), aminoterminal propeptide of type I collagen (PINP) and $\beta$-cross-linked carboxyterminal telopeptide of type I collagen $(\beta-\mathrm{CTx})\}, 2$ biochemical variables \{alkaline phosphatase (AKP) and Serum calcium $\}$ and 4 tumor markers (CEA, CA125, CA153 and CA199). These variables were selected because they were of potential clinical importance as indicated by a panel of experts. A number of data transformation techniques have been used to format and prepare the patient records to be processed by the learning algorithms (Table 1).

\section{Construction of the prediction models}

In this study, SPSS $19^{\circledR}$ and SPSS Modeler $14.1^{\circledR}$ (IBM, Armonk, NY, USA) were used to construct the DT, SVM and LR models. A $p$-value $\leq 0.05$ was considered to be significant for inclusion into the model. To validate each prediction model, we used a 10 -fold cross validation. In 10 -fold cross-validation, the data set is divided into 10 folds with equal size. Then training is carried out with 9 and testing with 1 ; the process is repeated until all parts have been tested.

A binary LR was performed to determine the data set under consideration, associates each record (a patient) with the probability of SREs. Stepwise selections of the independent variables were stepwise incremented and the corresponding coefficients were computed.

We constructed the DT as classification and regression trees (CART). The approach builds a binary tree by splitting the records at each node according to a function of a single input field. The evaluation function used for splitting in CART is the Gini index [23]. One of the most critical problems in tree construction is determining an appropriate size of tree. Standard methods use a "stopping rule" to determine appropriate tree sizes.

We used SVM with radial basis function (RBF) as kernels. The "SVM" function in SPSS Modeler was used to build our SVM model with the radial basis function kernel applied as its classification method.

\section{Comparison between prediction models}

Comparisons among LR, DT and SVM discrimination for all models were performed. Sensitivity, specificity, positive predictive value (PPV), negative predictive value (NPV), and accuracy were adopted to evaluate the performance of a model. Area under curve (AUC) was calculated to test the ability of each model to distinguish patients.

\section{Statistical analysis}

Patients were categorized into with SREs and without SREs. Qualitative variables were expressed by number, percent and compared by chi square or fishe's exact test. Quantitative variables were expressed by mean and standard deviation (SD) and compared by t student. Sensitivity, specificity, PPV, NPV and accuracy were calculated subsequently.

\section{ACKNOWLEDGMENTS AND FUNDING}

This study was supported by National Natural Science Foundation of China Grant (81201628). 


\section{CONFLICTS OF INTEREST}

The authors have declared no conflicts of interest.

\section{REFERENCES}

1. Coleman RE. Clinical features of metastatic bone disease and risk of skeletal morbidity. Clin Cancer Res. 2006; $12: 6243 \mathrm{~s}-6249 \mathrm{~s}$.

2. Coleman RE. Metastatic bone disease: Clinical features, pathophysiology and treatment strategies. Cancer Treat Rev. 2001; 7:165-176.

3. Cook RJ, Major P. Methodology for treatment evaluation in patients with cancer metastatic to bone. J Natl Cancer Inst. 2001; 93:534-538.

4. Kosteva J, Langer C. The changing landscape of the medical management of skeletal metastases in nonsmall cell lung cancer. Curr Opin Oncol. 2008; 20:155-161.

5. Coleman RE. Skeletal complications of malignancy. Cancer. 1997; 80:1588-1594.

6. Coleman RE. Bisphosphonates: Clinical experience. Oncologist. 2004; 9 suppl 4:14-27.

7. Vogel CL, Yanagihara RH, Wood AJ, Schnell FM, Henderson C, Kaplan BH, Purdy MH, Orlowski R, Decker JL, Lacerna L, Hohneker JA. Safety and pain palliation of zoledronic acid in patients with breast cancer, prostate cancer, or multiple myeloma who previously received bisphosphonate therapy. Oncologist. 2004; 9: 687-695.

8. Lipton A, Theriault RL, Hortobagyi GN, Simeone J, Knight RD, Mellars K, Reitsma DJ, Heffernan M, Seaman JJ. Pamidronate prevents skeletal complications and is effective palliative treatment in women with breast carcinoma and osteolytic bone metastases: long term follow-up of two randomized, placebo-controlled trials. Cancer. 2000; 88:1082-1090.

9. Saad F, McKiernan J, Eastham J. Rationale for zoledronic acid therapy in men with hormone-sensitive prostate cancer with or without bone metastasis. Urol Oncol. 2006; 24: 4-12.

10. Rosen LS, Gordon D, Tchekmedyian NS, Yanagihara R, Hirsh V, Krzakowski M, Pawlicki M, De Souza P, Zheng M, Urbanowitz G, Reitsma D, Seaman J. Long-term efficacy and safety of zoledronic acid in the treatment of skeletal metastases in patients with non-small cell lung carcinoma and other solid tumors: a randomized, Phase III, double-blind, placebo-controlled trial. Cancer. 2004; 100: 2613-2621.

11. Nørgaard M, Jensen AØ, Jacobsen JB, Cetin K, Fryzek JP, Sørensen HT. Skeletal related events, bone metastasis and survival of prostate cancer: a population based cohort study in Denmark (1999 to 2007). J Urol. 2010; 184:162-167.

12. Yong M, Jensen AÖ, Jacobsen JB, Nørgaard M, Fryzek JP, Sørensen HT. Survival in breast cancer patients with bone metastases and skeletal-related events: a population-based cohort study in Denmark (1999-2007). Breast Cancer Res Treat. 2011; 129:495-503.

13. Weinfurt KP, Castel LD, Li Y, Timbie JW, Glendenning GA, Schulman KA. Health-related quality of life among patients with breast cancer receiving Zometa or pamidronate disodium for metastatic bone lesions. Med Care. 2004; 42:164-175.

14. Weinfurt KP, Li Y, Castel LD, Saad F, Timbie JW, Glendenning GA, Schulman KA. The significance of skeletal-related events for the healthrelated quality of life of patients with metastatic prostate cancer. Ann Oncol. 2005; 16:579-584.

15. Costa L, Badia X, Chow E, Lipton A, Wardley A. Impact of skeletal complications on patients' quality of life, mobility, and functional independence. Support Care Cancer. 2008; 16:879-889.

16. Lee SK, Son YJ, Kim J, Kim HG, Lee JI, Kang BY, Cho HS, Lee S. Prediction model for Health-Related Quality of life of elderly with chronic diseases using Machine LearningTechniques. Healthc Inform Res. 2014; 2:125-134.

17. Hosseinzadeh F, Kayvanjoo AH, Ebrahimi M, Goliaei B. Prediction of lung tumor types based on protein attributes by machine learning algorithms. Springer Plus. 2013; 2: $1-14$.

18. Dreiseitl S, Ohno-Machado L. Logistic regression and artificial neural network classification models: a methodology review. J Biomed Inform. 2002; 35:352-359.

19. Salama GI, Abdelhalim M, Zeid MA-e. Breast Cancer Diagnosis on Three Different Datasets using Multiclassifiers. International Journal of Computer and Information Technology. 2012; 1:36-43.

20. Soloway MS, Hardeman SW, Hickey D, Raymond J, Todd B, Soloway S, Moinuddin M. Stratification of patients with metastatic prostate cancer based on extent of disease on initial bone scan. Cancer. 1988; 16:195-202.

21. Frankel HL, Hancock DO, Hyslop G, Melzak J, Michaelis LS, Ungar GH, Vernon JD, Walsh JJ. The value of posturalreduction in the initial management of closed injuries of the spine with paraplegia and tetraplegia. Paraplegia. 1969; 7:179-192.

22. Mirels H. Metastatic disease in long bones: a proposed scoring system for diagnosing impending pathological fractures. Clin Orthop. 1989; 249:256-264.

23. Zekic-Susac M, Pfeifer S, Durdevic I. Classification of entrepreneurial INTENTIONS by neural networks, decision trees and support vector machines. Croatian Operational Research Review. 2010; 1:62-71.

24. Lee SK, Kang BY, Kim HG, Son YJ. Predictors of medication adherence in elderly patients with chronic diseases using support vector machinemodels. Healthc Inform Res. 2013; 1:33-41.

25. Swets JA. Measuring the accuracy of diagnostic systems. Science. 1988; 240:1285-1293. 
26. Son MS, Moon PS. Big data era of Korea, if you do not want to Galapagos. Seoul: LG Business Insight. 2012 Available from: http://www.lgeri.com/uploadFiles/ko/pdf/ind/ LGBI1188-02_20120313130223.pdf Accessed Mar 2014.

27. Lim SJ, Min OK. Machine learning technology trends for big data processing. Electron Telecommun Trends. 2012; 27:55-63.

28. Duda, RO, Hart, PE, Stork, DG. Pattern Classification. 2. John Wiley and Sons. 2000.

29. Breiman L. Statistical Modeling: The Two Cultures. Statistical Science. 2001; 16:199-231.

30. Cook EF, Goldman L. A symmetric stratification. An outline for an efficient method for controlling confounding in cohort studies. Am J Epidemiol. 1988; 127:626-639.
31. Wang Z, Zhen Y, Wu C, Li H, Yang Y, Shen Z, Zhao H, Yao Y. CT fluoroscopy-guided percutaneous osteoplasty for the treatment of osteolytic lung cancer bone metastases to the spine and pelvis. J Vasc Interv Radiol. 2012; 23:1135-1142.

32. Wang Z, Qiao D, Lu Y, Curtis D, Wen X, Yao Y, Zhao H. Systematic literature review and network meta-analysis comparing bone-targeted agents for the prevention of skeletal-related events in cancer patients with bone metastasis. Oncologist. 2015; 20:440-449. 